\title{
Condiciones facilitadoras para el desarrollo del emprendimiento: una mirada al caribe colombiano
}

\author{
Karelis Del C. Barrios-Hernández*, Brenda C. Figueroa-Saumet, María J. Niebles-Bárcenas, y \\ Robinson De J. Palacio-Pérez \\ Facultad de Administración y Negocios, Universidad Simón Bolívar, Barranquilla-Colombia \\ (correo-e: kbarrios22@unisimonbolivar.edu.co; karelisbarrios03@gmail.com; brenda.figueroa@unisimon.edu.co; \\ mniebles8@unisimon.edu.co; rpalacio11@unisimon.edu.co) \\ * Autor a quien debe dirigirse la correspondencia
}

Recibido May. 15, 2021; Aceptado Jul. 13, 2021; Versión final Sept. 14, 2021, Publicado Feb. 2022

\begin{abstract}
Resumen
El objetivo de este estudio es identificar las condiciones facilitadoras para la gestión del emprendimiento en el caribe colombiano. Se desarrolla una investigación cuantitativa que involucra un cuestionario de 20 ítems medidos en una escala Likert de siempre a nunca, aplicado a 409 emprendedores ubicados en la ciudad de Barranquilla (Colombia) con dos o más años de funcionamiento. Por el carácter empírico del estudio, se recurre a un análisis factorial. Los resultados revelan cinco condiciones facilitadoras para la gestión del emprendimiento: 1) la relevancia del diseño de modelo negocio, 2) la educación financiera, 3) la planeación, 4) el direccionamiento estratégico y 5) el conocimiento del entorno. Se concluye que estos son componentes claves para la permanencia del emprendimiento en el mercado y que en la medida que los emprendedores estén preparados para anticiparse a las condiciones del entorno o adaptarse rápidamente a los cambios, sus negocios podrán permanecer en el tiempo.
\end{abstract}

Palabras clave: emprendimiento; modelo; negocio; educación financiera; planeación; direccionamiento; conocimiento; entorno

\section{Facilitating components for the management of entrepreneurship: a look at the Colombian Caribbean}

\begin{abstract}
The primary objective of this study is to identify the main facilitating components for entrepreneurial management in the Colombian Caribbean. The research methodology is quantitative and involves using a 20item questionnaire measured on a Likert scale (always to never). The surveyed sample is composed of 406 entrepreneurs, with two or more years of experience, from the city of Barranquilla (Colombia). A factor analysis is performed due to the empirical nature of the study. The results revealed five facilitating components for entrepreneurial management: 1) the business model's design relevance, 2) financial education, 3) planning, 4) strategic direction, and 5) business environment knowledge. It is concluded that these five components are key for lifelong entrepreneurial businesses and that as long as entrepreneurs are prepared to foresee and quickly adapt to changes in their surrounding environments their businesses will persist over time.
\end{abstract}

Keyword: entrepreneurship; business model; financial education; strategic planning; direction; knowledge; environment 


\section{INTRODUCCIÓN}

El emprendimiento es uno de los motores de crecimiento, transformación y desarrollo de los países (Terjesen, y Hessels 2009) El término fue utilizado por primera vez en el siglo XVIII, tiempo después muchos autores se interesaron por desarrollar diferentes definiciones con respecto al tema. Suárez (2014) concibe el emprendimiento como una relación entre la actitud, cultura y capacidad personal de crear o desarrollar negocios con una alta dosis de creatividad que responde a situaciones del mercado. En este sentido, el emprendimiento consiste en poner en marcha una actividad económica a través de la producción de bienes o servicios para satisfacer las necesidades de una población o aprovechar oportunidades del entorno (Gutiérrez et al., 2017).

En Colombia fue creada la ley del emprendimiento, la cual busca apoyar a los emprendedores independientemente del tamaño de los negocios, con esta se busca brindar una ruta normativa que contribuya a la creación negocios, con el fin de incentivar la generación de empleos y tejido empresarial en los territorios. Así mismo, desde el año 2006, se promueve el fomento de la cultura de emprendimiento desde el sistema educativo, Ley 1014 del año 2006. Esta ley define el emprendimiento como una forma de pensar y proceder, encaminada hacia la consecución de recursos económicos para aprovechar oportunidades. Por eso, se ha relacionado el emprendimiento con el progreso, apoyando la solución de problemas generados por el desempleo y pobreza para lo cual se requiere de la influencia de las instituciones educativas, especialmente la Universidad (Cárdenas et al., 2015).

Por tanto, para que el emprendimiento apoye la resolución de problemas en las regiones, requiere que se genere a partir de procesos innovadores que involucren la interacción entre universidad, estado, sociedad y el sector empresarial. De esta manera, se establece contacto efectivo con los actores del entorno mejorando la competitividad. Es allí donde esta relación juega un rol preponderante para la generación de emprendimientos de gran impacto en las regiones (Barrios-Hernández y Olivero-Vega, 2020), para que los proyectos respondan con soluciones prácticas e innovadoras. Por tanto, no se trata de relacionarse con el sector externo de manera independiente, sino de construir verdaderos entornos de colaboración (BarriosHernández et al., 2017) para generar y fomentar el desarrollo de competencias que despierten la creatividad e innovación (Salinas y Osorio-Bayter 2012).

En este trabajo se aborda las condiciones facilitadoras para el desarrollo del emprendimiento en el caribe colombiano, partiendo del hecho que la acción de emprender está encontrando una creciente aceptación social y, cada vez más, mejores incentivos económicos de parte del gobierno. Así mismo, la consolidación del ecosistema emprendedor en Colombia se ha visto favorecida por el éxito de startups, que han superado los obstáculos propios del emprendimiento para crear proyectos con alto potencial de escalabilidad. Por otra parte, este artículo cobra real importancia en la actualidad, debido a que con la llegada del COVID 19 se ha impulsado el cambio de la mentalidad de los emprendedores quienes hoy están buscando fortalecer sus negocios con procesos digitales que le permitan adaptarse a las nuevas condiciones del mercado.

Es por eso que, entender el mercado, rediseñar los modelos de negocios bajo una perspectiva de mentalidad digital, educar financieramente a los emprendedor, planear y direccionar a los emprendimientos se convierte en un reto para alcanzar no solo su sostenibilidad sino su escalabilidad. Por un lado, el modelo de negocio supone la forma como una organización crea y captura el valor para sus Stakeholders (Osterwalder y Pigneur, 2010). Lo que indica que tener un modelo de negocio validado en el mercado requiere de una planeación, debido a que la velocidad y ritmo con la que se están produciendo cambios tanto al interior de la organización, como en su entorno, trayendo como consecuencia que los objetivos estratégicos sean cada vez menos efectivos, ya que estos están dirigidos a solucionar debilidades y amenazas inminentes y predecibles, dejando a un lado las posibilidades y oportunidades que presenta el entorno y que requieren total atención de la dirección(Consuegra y Ariza, 2016). Además, parte de las debilidades identificadas en los emprendimientos, se refieren a la carencia de formación académica en temas técnicos y administrativos de sus lideres, entonces por más que las ideas de negocio sean escalables, terminan absorbiendo costos innecesarios debidos a la falta de un aprendizaje previo, que sumados a los cambios del entorno no permiten anticiparse mediante la toma acertada de decisiones. El propósito de este articulo es enriquecer la literatura sobre las condiciones facilitadoras para el desarrollo del emprendimiento y ha sido estructurado en 5 sesiones. Primero, una introducción. Seguidamente, en la segunda, la fundamentación teórica. En la tercera, el diseño metodológico. En la cuarta sesión, se encuentran los resultados y finalmente, en la quinta, las conclusiones.

\section{OTROS ANTECEDENTES}

El emprendimiento es un término que, aunque ha sido usado a lo largo de la historia, solo en los últimos años es donde más auge ha tenido, y en realidad esto se ha dado por las condiciones del entorno dadas a la 
presencia del COVID 19 y el incremento de las tasas de desempleo. Una de las primeras menciones al emprendimiento del que hay data, es a través de Cantillon (1755) quien hace referencia al emprendedor con el término de entrepreneur, referenciándolo como un hombre racional por excelencia, desenvolviéndose en la sociedad mercantil donde la toma de decisiones se realizaba en base a probabilidades inciertas, teniendo en cuenta la competencia e incertidumbre como valor constante, de igual forma lo definió como el individuo que compra a ciertos precios y los combina en forma lógica para obtener de allí un nuevo producto.

En el siglo XX, Schumpeter (1942) desde su aporte como economista, dijo que para que el sistema económico se desarrollara eran necesarias las ganancias que provenían del cambio que generaba de forma constante el emprendedor a través de sus negocios; por lo cual esta era la base o punto de inicio del sistema económico. De esta forma el emprendedor, a raíz de dichos negocios o innovaciones, las cuales manejaba de forma dinámica, adaptándose al cambio, tenía la capacidad de causar el desequilibrio del mercado. De acuerdo con Duarte y Ruiz Tibana (2009) un emprendedor se identifica porque es capaz de realizar algo novedoso e innovador, así como también de dar uso a algo que ya existe y que de esta manera pueda contribuir al mejoramiento de su propia vida y la de su alrededor, el emprendedor es aquel que genera ideas nuevas, y además es capaz de convertir un problema en una oportunidad. Valls, et al. (2019) dice que emprendimiento es la capacidad de comenzar y crear un proyecto, a través de identificar ideas y oportunidades, evaluado factores externos económicos, políticos, sociales y ambientales, pero también internos como talento humano, infraestructura y recursos financieros. Las personas emprendedoras son aquellas que tienen capacidad de liderazgo y actitud proactiva, capaces de generar alternativas ante situaciones de cualquier índole (Valls, et al., 2019).

En Colombia, el emprendimiento ha ocupado un lugar destacado en la estrategia de reactivación económica, específicamente, se ha constituido como uno de los pilares de desarrollo del nuevo gobierno, a través del fortalecimiento de las instituciones gubernamentales, al igual que el apoyo del ecosistema conformado por centros de innovación, cámaras de comercio, universidades, entre otros. Sin embargo, las medidas de bioseguridad implementadas para la contención del COVID-19 trajo consigo un distanciamiento social que está afectando a los emprendedores, por una parte, debido a la disminución de la demanda de bienes y servicios (Kantis y Angelelli, 2021) y por la otra a la falta de adaptación rápida de los emprendedores a los cambios del entorno. Entre enero y diciembre del año 2020 hubo una disminución del $211 \%$ en la creación de nuevas empresas. Pasando de 309.463 en el 2019 a 99.461 creaciones en el 2020. Específicamente, un $14 \%$ de estas últimas se crearon en Barranquilla y Cartagena, con 8.260 empresas y 5.551 empresas respectivamente (Portafolio, 2021).

De esta manera, el reto para el país no es solo contener el COVID 19 con las medidas sanitarias adecuadas, si no paralelamente reactivar la economía para afectar lo menos posible a las empresas. En Barranquilla, la Unidad de Apoyo al Empresario de la Alcaldía Distrital de Barranquilla y operada por la Universidad Simón bolívar fue la encargada de desarrollar un programa para reactivación económica, segura y responsable. Si bien, esta Unidad existe desde el año 2016, a unido esfuerzo de la triada Universidad- Empresa- Estado (Barrios-Hernández y Olivero-Vega, 2020) para contribuir a generar la sostenibilidad de las organizaciones a través de un permanente crecimiento y mejorar los indicadores de competitividad, simultáneamente a un incremento en el bienestar social y en las condiciones de calidad de vida de los habitantes de la ciudad (Consuegra et al., 2017). Así mismo, el programa CREEce de Cámara de Comercio, en el cual también participó el Centro de Crecimiento Empresarial MacondoLab de la Universidad Simón Bolívar, contribuyó al fortalecimiento empresarial para mejorar la oferta de valor de negocios de diferentes sectores con un enfoque de formalización. Ambos programas han resultado un apoyo para la gestión de condiciones que faciliten el desarrollo de los emprendimientos de la ciudad y su adaptación a la nueva realidad.

Por una parte, Kreiser et al., (2010) uno de los factores que influyen en el éxito de los emprendimientos es la cultura. Además, cada vez son más los estudios que afirman que un cambio en la educación de la sociedad, desde la etapa escolar hasta la universidad, puede generar una alta intención emprendedora y por ende desarrollo de emprendimiento. Por la otra, la financiación del emprendimiento contribuye a su sostenibilidad en el tiempo, donde los amigos y familiares del emprendedor, se constituyen como su principal fuente (Consuegra, et al., 2017). Es por ello, que la educación financiera juega un papel fundamental en el desarrollo de los emprendimientos debido que les permite adquirir mayores conocimientos sobre riesgos de las operaciones financieras, creando confianza al momento de toma de decisiones en búsqueda de fuentes de financiación que le permitan mejorar el bienestar económico (Romero, et al., 2021).

Sin embargo, estos factores no son los únicos que según la literatura contribuyen al desarrollo de emprendimiento. Autores como Osterwalder y Pigneur (2010) mencionan que para que un emprendimiento disminuya su riesgo debe entender la forma como crea y captura el valor a través del modelo de negocio. En el cual entender el entorno y las oportunidades que este se encuentran juegan un papel fundamental. Ya que parte del conocimiento de un mercado con unas características y perfiles particulares, así mismo, plantean 
como realizar una buena estructura y comprender la naturaleza de los modelos de negocio explicando cómo posicionar el modelo en un panorama muy competitivo.

Desde otra perspectiva, Igor Ansoff, indica que cuando una empresa busca oportunidades de crecimiento, dispone de cuatro opciones para lograrlo: penetración y desarrollo de mercado, lanzamiento y diversificación de productos (Ansoff, 1976). Por tanto, la planeación y el direccionamiento facilitan la creación de negocios innovadores a partir de entender y anticiparse al entorno. Sin embargo, teniendo en cuenta que el entorno tiene un comportamiento que no se puede predecir en su totalidad, así también sucede con los resultados de los negocios, porque la aleatoriedad se trasmite a ellos y la estrategia sirve solo como mecanismo amortiguador del riesgo. Cuando las condiciones del entorno cambian constantemente y a gran velocidad, las decisiones estratégicas van perdiendo su validez a corto plazo, por lo que el cambio de estrategia se debe hacer con mayor frecuencia y rapidez. Es por eso que, a medida que el entorno competitivo se acelera, la probabilidad que una estrategia pueda seguir siendo efectiva, sin importar lo bien que se haya pensado, disminuye proporcionalmente (Davis y Meyer, 1998) obligando a los negocios a estudiar el entorno en su día a día.

\section{METODOLOGÍA}

En este artículo se plantea un tipo de investigación cuantitativa cuya metodología de análisis consta de 2 componentes estadísticos: análisis preliminar en SPSS y análisis factorial (AF) tal como se evidencia en la figura 1. El instrumento que se escogió para la recolección de los datos es un cuestionario que consta de 20 ítems, el cual fue aplicado a 409 emprendedores con 2 o más años de gestación de su negocio. Antes de su formulación definitiva se elaboró un cuadro metodológico con el propósito de establecer las variables a medir con sus respectivos indicadores.

Para este cuestionario se utilizó una escala tipo Likert (Siempre, casi siempre, algunas veces, pocas veces y nunca). Este tipo de escala se corresponde con un nivel de medición ordinal que consiste en una serie de juicios ante los cuales se solicita la reacción del sujeto y que representa lo que se está interesado en medir (Barrios-Hernández., 2017). Previo a la aplicación, este instrumento fue enviado a 4 expertos en emprendimiento, los cuales manifestaron sus apreciaciones sobre ellos y en los casos necesarios se realizaron los ajustes, en estos fue determinante aspectos como la brevedad y claridad de los ítems. Así mismo para la construcción del instrumento se realizó una revisión exhaustiva de la literatura sobre emprendimiento. Las fuentes consultadas en su mayoría fueron artículos científicos y documentos de bases de datos como Scielo, WOS y Scopus.

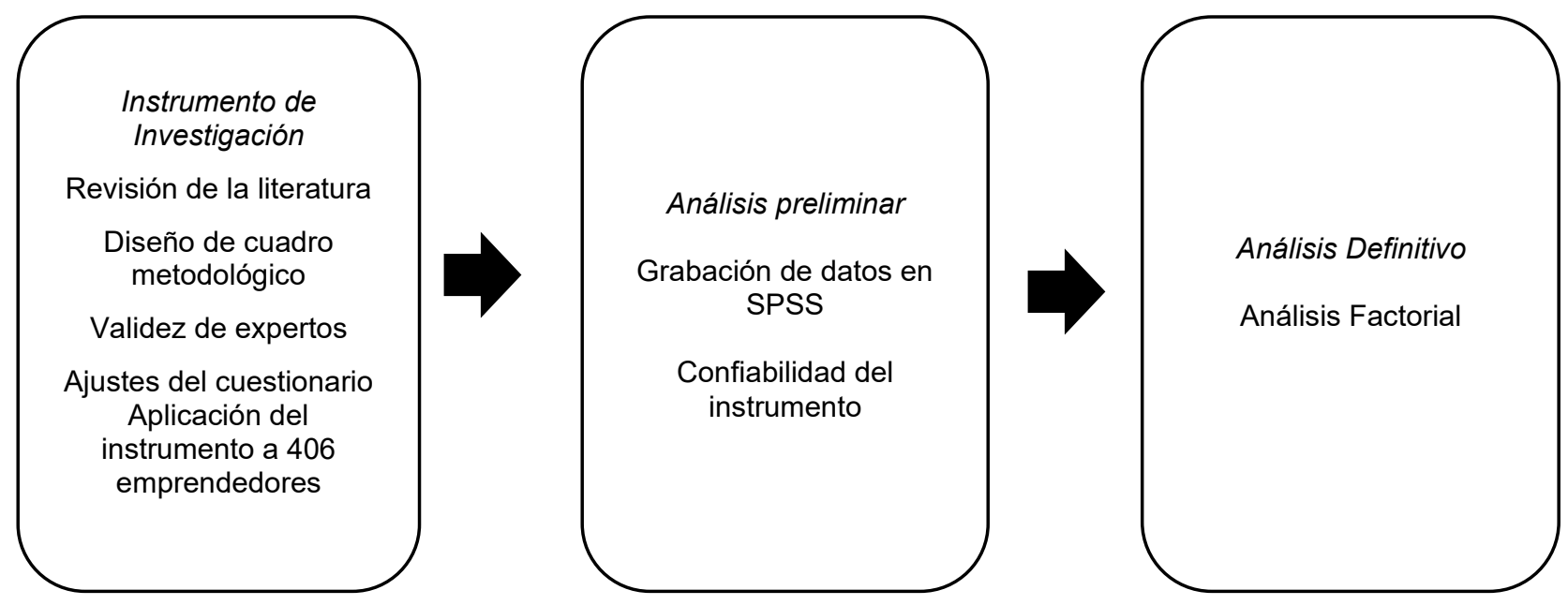

Fig. 1: Etapas metodológicas de la investigación.

Por una parte, el término AF se refiere a un conjunto de técnicas estadísticas y a un método único de interdependencia (Khan, 2006), que se emplea con el objeto de reducir un gran número de variables operativas en un número inferior de variables conceptuales. Se puede afirmar que el principal objetivo del AF es la reducción de un número de variables originales, mediante la obtención de factores que son variables latentes no observadas. Estos factores pueden considerarse componentes que agrupan variables que se correlacionan en una alta proporción. Por tanto, el AF cobra sentido exclusivamente cuando se demuestra la presencia de variables que están correlacionadas. El resultado definitivo del AF pretende demostrar la la 
unidimensionalidad, es decir una variable se relaciona exclusivamente con un factor, la fiabilidad y la validez de los factores con los que se miden las condiciones facilitadoras para el desarrollo de emprendimiento (Hattie, 1985; Bohrnstedt,1876; Acosta, 2010). A continuación, ha sido necesario perfeccionar este nuevo conjunto de factores con el propósito de validar su estructura, garantizando la unidimensionalidad de los factores, la fiabilidad, así como la validez convergente y discriminante (Acosta, 2010), con el objeto de llegar a unos factores significativos (MacCallum, 1986; Acosta, 2010).

\section{RESULTADOS}

El primer paso del análisis metodológico fue hacer las pruebas en el paquete estadístico SPSS que permiten en primera instancia medir la fiabilidad del cuestionario y en segunda si el instrumento nos permite hacer el AF. Para la fiabilidad del instrumento se utilizó como medida estadística el alfa de Cronbach cuyo resultado en la aplicación de la muestra total de 406 emprendedores fue de 0,89 tal como se evidencia en la tabla 1 que, evaluado con los criterios de George y Mallery, 1995, la fiabilidad del instrumento es buena, es decir, evalúa con certeza el constructo que pretende medir, que para esta investigación es la gestión del emprendimiento en función de sus condiciones facilitadoras.

Tabla 1. Procesamiento de datos

\begin{tabular}{|l|l|c|c|}
\hline \multicolumn{2}{|c|}{ Resumen de procesamiento } & N & $\%$ \\
\hline \multirow{3}{*}{ Casos } & Válido & 409 & 100 \\
\cline { 2 - 4 } & Excluido & 0 & 0 \\
\cline { 2 - 4 } & Total & 409 & 100 \\
\hline \multirow{2}{*}{ Estadísticas de fiabilidad } & Alfa de Cronbach & N & $\%$ \\
\cline { 2 - 4 } & 0,89 & 20 & 100 \\
\hline
\end{tabular}

El siguiente paso es el cálculo de los estadísticos de la regla de Káiser-Meyer-Olkin (KMO) y la prueba de esfericidad de Bartlett, las cuales se presentan en la tabla 2. La medida de KMO dio como resultado 0,877, lo que indica que el resultado es muy notable para realizar AF. Así mismo, la prueba de esfericidad de Bartlett dio un Sig. (p-valor) de 0.000 lo que indica que rechazamos la hipótesis nula de que no existe correlación entre las variables, es decir, si se puede realizar el AF (Acosta, 2010).

Tabla 2. Prueba de KMO y Bartlett

\begin{tabular}{|l|l|l|}
\hline \multicolumn{2}{|l|}{ Medida Kaiser-Meyer-Olkin de adecuación de muestreo } & 0,877 \\
\hline \multirow{2}{*}{ Prueba de esfericidad de Bartlett } & Aprox. Chi-cuadrado & 4342,333 \\
\cline { 2 - 4 } & $\mathrm{gl}$ & 190 \\
\cline { 2 - 4 } & Sig. & 0 \\
\hline
\end{tabular}

Para comenzar agrupar los factores o componentes de este análisis, se ha iniciado con la matriz de correlaciones. Seguidamente, para la retención del número de componentes principales o factores comunes (comunalidades) se ha aplicado el método de extracción: análisis de componentes principales a las variables del cuestionario. El criterio de decisión para retener es aquellos factores con un autovalor o valor propio superior a 1 (Acosta, 2010). En la tabla 3 se observan los resultados obtenidos de la extracción. Un total de 5 componentes o factores comunes retenidos cuyo valor propio es superior a 1. En el AF, el primer componente modelo de negocio tiene un valor propio de 4,375 y su explicación de la varianza de las variables es del $21,875 \%$. El segundo componente educación financiera explica la varianza en $15,277 \%$, el tercer componente planeación en un $11,83 \%$, el cuarto componente direccionamiento explica el objeto de estudio en un $10,569 \%$, el quinto componente entorno un $8,294 \%$, respectivamente. Los 5 componentes o factores explican el $67,844 \%$ la varianza explicada. 
Tabla 3. Análisis Factorial varianza total explicada.

\begin{tabular}{|c|r|r|r|r|r|r|r|r|r|}
\hline Componente & \multicolumn{1}{|c|}{ Total } & $\begin{array}{c}\text { \% de } \\
\text { varianza }\end{array}$ & $\begin{array}{c}\text { \% } \\
\text { acumulado }\end{array}$ & Total & $\begin{array}{c}\text { \% de } \\
\text { varianza }\end{array}$ & $\begin{array}{c}\text { \% } \\
\text { acumulado }\end{array}$ & Total & $\begin{array}{c}\% \text { de } \\
\text { varianza }\end{array}$ & $\begin{array}{c}\% \\
\text { acumulado }\end{array}$ \\
\hline 1 & 7,158 & 35,791 & 35,791 & 7,158 & 35,791 & 35,791 & 4,375 & 21,875 & 21,875 \\
\hline 2 & 2,601 & 13,003 & 48,794 & 2,601 & 13,003 & 48,794 & 3,055 & 15,277 & 37,152 \\
\hline 3 & 1,568 & 7,839 & 56,633 & 1,568 & 7,839 & 56,633 & 2,366 & 11,83 & 48,982 \\
\hline 4 & 1,223 & 6,113 & 62,746 & 1,223 & 6,113 & 62,746 & 2,114 & 10,569 & 59,551 \\
\hline 5 & 1,02 & 5,099 & 67,844 & 1,02 & 5,099 & 67,844 & 1,659 & 8,294 & 67,844 \\
\hline
\end{tabular}

Las variables que conforman cada uno de los componentes en el AF y que son las que presentan mayores asociaciones, medidas por los coeficientes de correlación o cargas factoriales se presentan en la tabla 4.

Tabla 4. Análisis Factorial exploratorio (AFE) Comunalidades - extracción de componentes

\begin{tabular}{|c|c|c|c|}
\hline $\begin{array}{l}N^{\circ} \text { de } \\
\text { items }\end{array}$ & Comunalidades & Inicial & Extracción \\
\hline 1 & $\begin{array}{l}\text { El emprendedor identifica el margen de utilidad en su ejercicio } \\
\text { comercial. }\end{array}$ & 1 & 0,677 \\
\hline 2 & $\begin{array}{l}\text { El emprendedor reconoce el método adecuado para hallar el precio } \\
\text { de venta de sus productos o servicios. }\end{array}$ & 1 & 0,665 \\
\hline 3 & $\begin{array}{l}\text { El emprendedor identifica plenamente la estructura de costo de su } \\
\text { negocio }\end{array}$ & 1 & 0,779 \\
\hline 4 & $\begin{array}{l}\text { El emprendedor determina el punto de equilibrio antes de ofertar un } \\
\text { nuevo producto o servicio. }\end{array}$ & 1 & 0,664 \\
\hline 5 & $\begin{array}{l}\text { El emprendedor cumple con las obligaciones tributarias de su ejercicio } \\
\text { comercial }\end{array}$ & 1 & 0,568 \\
\hline 6 & $\begin{array}{l}\text { El negocio cuenta con recursos financieros suficientes para sus } \\
\text { operaciones }\end{array}$ & 1 & 0,532 \\
\hline 7 & $\begin{array}{l}\text { El negocio cuenta con recursos físicos y tecnológicos suficientes para } \\
\text { su ejercicio comercial }\end{array}$ & 1 & 0,504 \\
\hline 8 & Tiene un modelo de negocio validado en el mercado & 1 & 0,692 \\
\hline 9 & Cuenta con un factor diferenciador atractivo al mercado & 1 & 0,532 \\
\hline 10 & $\begin{array}{l}\text { El emprendimiento mantiene un factor diferencial de su negocio frente } \\
\text { a sus competidores. }\end{array}$ & 1 & 0,568 \\
\hline 11 & $\begin{array}{l}\text { El emprendedor presta especial atención al posicionamiento de su } \\
\text { marca en el mercado }\end{array}$ & 1 & 0,544 \\
\hline 12 & Los canales utilizados para la publicidad son los adecuados & 1 & 0,534 \\
\hline 13 & $\begin{array}{l}\text { Los canales de ventas son satisfactorios entregando resultados } \\
\text { acorde a la inversión realizada }\end{array}$ & 1 & 0,564 \\
\hline 14 & La marca del negocio es reconocida en el mercado & 1 & 0,764 \\
\hline 15 & Detecta sus oportunidades y sabe como aprovecharlas & 1 & 0,749 \\
\hline 16 & $\begin{array}{l}\text { La visión ha sido formulada acorde a un estudio del entorno en el que } \\
\text { se desenvuelve el negocio }\end{array}$ & 1 & 0,868 \\
\hline 17 & $\begin{array}{l}\text { El emprendedor ha formulado estrategias para el cumplimiento de la } \\
\text { visión }\end{array}$ & 1 & 0,856 \\
\hline 18 & La misión ha sido definida de forma correcta & 1 & 0,898 \\
\hline 19 & Tiene metas definidas para su cumplimiento en el corto plazo & 1 & 0,769 \\
\hline 20 & $\begin{array}{l}\text { Los objetivos que desea alcanzar son coherentes con las estrategias } \\
\text { implementadas }\end{array}$ & 1 & 0,843 \\
\hline
\end{tabular}


Dada la varianza explicada de componentes en la que resulto el AF y las cargas factoriales de los mismos y con el propósito de lograr una mejor interpretación de los factores y lograr la unidimensionalidad de las variables que los componen se procedió a aplicar la técnica de rotación Varimax con normalización Kaiser. Al aplicar las comunalidades para la extracción por medio del análisis de componentes principales tenemos que los 5 factores extraídos permanecen dado que todos mantienen un autovalor o valor propio superior a 1, En el paquete estadístico SPSS el método de componentes principales es iterativo. Este proceso llega a su fin cuando la comunalidad estimada para cada variable original entre dos iteraciones sucesivas es menor de 0,001. La convergencia para este caso se dio en 7 iteraciones. Para tener una mejor interpretación de los competentes se ha aplicado la técnica de rotación ortogonal por medio del método Varimax con normalización Kaiser, se observa en la tabla 5 el nombre del factor, las variables que lo componen y la carga factorial.

Adicionalmente, se evidencia que hay variables que cargaron en más de un factor. El criterio estadístico, para tomar la decisión de eliminar la variable en uno o ambos componentes, es el de la diferencia entre la carga factorial del mayor y el menor: Si la diferencia es inferior a 0,1 se eliminará la variable de ambos componentes dado que no hay claridad para su interpretación, pero si la diferencia es mayor o igual de 0,1 se eliminará la variable del factor donde esta tenga la menor carga factorial dado que es significativa para el componente donde tiene la mayor carga factorial. En este caso las variables 5 es eliminada al no mostrar claridad para su interpretación porque su carga factorial es inferior a 0,5 en dos factores.

Tabla 5. Matriz de Componente Rotado.

\begin{tabular}{|c|c|c|c|c|c|c|}
\hline Factor & $\begin{array}{c}N^{\circ} \text { de } \\
\text { Variables }\end{array}$ & \multicolumn{5}{|c|}{ Cargas factoriales } \\
\hline \multirow{8}{*}{ Modelo de negocio } & 6 & 0,563 & 0,398 & 0,059 & 0,002 & 0,23 \\
\hline & 7 & 0,583 & 0,364 & $-0,072$ & 0,146 & 0,066 \\
\hline & 8 & 0,789 & 0,251 & 0,047 & 0,064 & 0,023 \\
\hline & 10 & 0,691 & $-0,044$ & 0,003 & $-0,039$ & 0,294 \\
\hline & 11 & 0,68 & 0,22 & 0,089 & 0,061 & 0,147 \\
\hline & 12 & 0,646 & 0,058 & 0,175 & 0,188 & 0,216 \\
\hline & 13 & 0,67 & 0,309 & 0,084 & 0,074 & 0,081 \\
\hline & 14 & 0,811 & 0,271 & 0,093 & 0,076 & 0,136 \\
\hline \multirow{4}{*}{ Educación financiera } & 1 & 0,338 & 0,735 & $-0,004$ & 0,136 & $-0,062$ \\
\hline & 2 & 0,325 & 0,747 & 0,009 & 0,009 & $-0,043$ \\
\hline & 3 & 0,322 & 0,801 & 0,078 & 0,014 & 0,168 \\
\hline & 4 & 0,04 & 0,694 & 0,14 & 0,247 & 0,315 \\
\hline \multirow{3}{*}{ Planeación } & 16 & 0,05 & 0,064 & 0,928 & 0,019 & 0,022 \\
\hline & 18 & 0,116 & 0,091 & 0,916 & 0,16 & 0,111 \\
\hline & 19 & 0,119 & 0,009 & 0,668 & 0,55 & 0,079 \\
\hline \multirow{2}{*}{ Direccionamiento } & 17 & 0,052 & 0,178 & 0,03 & 0,904 & 0,054 \\
\hline & 20 & 0,181 & 0,096 & 0,27 & 0,848 & 0,102 \\
\hline \multirow{2}{*}{ Entorno } & 5 & 0,144 & 0,436 & 0,225 & 0,282 & 0,476 \\
\hline & 15 & 0,248 & 0,066 & 0,064 & 0,016 & 0,824 \\
\hline
\end{tabular}

Interpretación de factores

El factor modelo de negocio está conformado por 8 ítems donde el mayor, la variable 14, tiene una carga factorial de 0,811 y se refiere a si la marca del negocio es reconocida en el mercado, el segundo (ítem 8) de este factor evalúa la existencia de un modelo de negocio validado en el mercado con una carga factorial de 0,789 , en tercera posición (ítem 10) se refiere al factor diferencial del emprendimiento frente a sus competidores con una carga factorial de 0,691, en el cuarto lugar (ítem 11) hace alusión a si el emprendedor presta especial atención al posicionamiento de su marca en el mercado con una carga factorial de 0,68. En la quinta y sexta posición (ítems 13 y12) hacen referencia a los canales utilizados para llevar la propuesta de valor al cliente con una carga factorial de 0,67 y 0,646 respectivamente. 
Finalmente, con igual medida de carga factorial de 0,583 encontramos los recursos financieros, físicos y tecnológicos para el desarrollo de las operaciones (ítems 6 y 7). Este factor, reafirma los postulados de la literatura existente donde Osterwalder y Pigneur (2010) expresan que las base para la creación y gestión de un emprendimiento es el diseño y validación de su modelo de negocio, porque con esto definen la forma como crean, proporcionan y capturan valor. Este modelo se compone de 9 bloques que incluye los ítems mencionados, tales como propuesta de valor, canales y recursos claves, entre otros.

El segundo factor denominado educación financiera, lo componen en primer lugar la capacidad que tiene el emprendedor para identificar plenamente la estructura de costo de su negocio(ítem 3) con una carga factorial de 0,801 , seguido de la facilidad del emprendedor para reconocer el método adecuado para hallar el precio de venta de sus productos o servicios (ítem 2) con una carga de 0,747. Así mismo, con una carga de 0,735 se valora en tercera posición la identificación por parte del emprendedor del margen de utilidad en su ejercicio comercial. En un cuarto lugar se ubica con una carga factorial de 0,694 si el emprendedor determina el punto de equilibrio antes de ofertar un nuevo producto o servicio. Igualmente, que el factor 1 , este confirma que una de las condiciones facilitadoras para la gestión del emprendimiento no es solo la cantidad de recursos financieros disponibles con los que cuenta, sino la forma como las finanzas son manejadas en el negocio. Según Correa, et al., (2010) la educación financiera es importante en el desarrollo de un emprendimiento, debido a que logra una integración de los componentes del plan de negocios traduciéndolos a términos cualitativos y cuantitativos. De esta manera, contribuye a desarrollar la capacidad de estimar las condiciones presentes y futuras al tiempo que apoya la cuantificación y cualificación de los riesgos asociados a la operación y toma de decisiones en cualquier compañía.

En el tercer factor, encontramos las variables relacionadas con la planeación. En primer lugar se ubica con una carga factorial 0,928 la variable relacionada con la gestión de la visión a partir del estudio del entorno en el que se desenvuelve el negocio (item16). Seguidamente, en la segunda ubicación la capacidad del emprendedor para definir la misión de forma correcta acorde a una planeación (ítem 18) con una carga factorial de 0,916 . Finalmente, la variable que permite identificar si el emprendedor mantiene metas definidas para su cumplimiento en el corto plazo (ítem 19) con una carga factorial 0,668.

El cuarto factor, denominado direccionamiento, se ubican dos variables. En la primera posición con una carga factorial de 0,904 si el emprendedor ha formulado estrategias para el cumplimiento de la visión (ítem 17), seguidamente la variable metas para el cumplimiento en el corto plazo (ítem 20) con una carga factorial de 0,848. Siguiendo a Correa et al., (2009) la mayoría de los emprendedores no realizan una planeación estratégica, por ende tampoco una planeación financiera, y en consecuencia una deficiente administración de sus recursos. Por tanto, es relevante que como una de las condiciones facilitadoras para el éxito de los emprendimientos y su permanencia en el tiempo se incluya acorde a los resultados de este estudio y a la teoría mencionada, la planeación.

El quinto factor, entorno, lo compone una variable relacionada con la capacidad del emprendedor de detectar sus oportunidades y saber como aprovecharlas con una carga factorial de 0,824 , lo cual va en concordancia con lo planteado por Teece (2009) quien afirma que las organizaciones deben mantenerse en constante alerta para detectar oportunidades y amenazas. Dentro de esto, juega un papel fundamental las percepciones individuales y relaciones internas, especialmente la del cuerpo directivo, el sistema de información y el sistema de conocimiento, pero también requiere de la construcción de entornos colaborativos (Barrios-Hernández et al., 2017). A partir de los resultados obtenidos y la literatura revisada se pueden evidenciarse cinco condiciones facilitadoras para la gestión del emprendimiento: modelo de negocio, recursos financieros, planeación, direccionamiento y conocimiento del entorno, las cuales en su conjunto han permitido la permanencia de 406 emprendimientos.

\section{CONCLUSIONES}

Siendo el emprendimiento una fuente para el desarrollo de las regiones para los gobiernos, el emprendimiento ha sido uno de los temas más renombrados en los últimos cinco años en Colombia, esto debido a que contribuye al diseño de planes, programas y proyectos de políticas públicas de creación de empresas y nuevos negocios, así mismo contribuyendo al crecimiento económico y a la generación de nuevos empleos. Esta investigación permitió evidenciar las siguientes tres conclusiones:

La primera, se refiera que el diseño de un modelo de negocio juega un papel preponderante en la gestión de emprendimiento y en la disminución del riesgo al fracaso, porque este incluye de manera integral la hoja de ruta que el negocio debe seguir. No obstante, este modelo no es estático, requiere el compromiso de los lideres para la construcción permanente y adaptación a las nuevas condiciones que pueda imponer el mercado. 
La segunda, la educación financiera constituyen pieza clave en la sociedad y la sostenibilidad de los negocios. El desconocimiento en temas financieros afecta significativamente la toma de decisiones y la intervención en procesos económicos. Por tal razón, se hace necesario desarrollar y poner en práctica la educación financiera en los emprendedores con el fin de desarrollar las habilidades y destrezas que se requiere para manejar sus productos financieros y la economía de sus empresas o negocios. Finalmente, la tercera se refiere a que la planeación, el direccionamiento estratégico y el conocimiento del entorno, las cuales constituyen la base fundamental para definir la razón de ser del emprendimiento y donde se visualiza en un futuro cercano. Por tanto, en la medida que los emprendedores estén preparados para anticiparse a las condiciones del entorno o adaptarse rápidamente a los cambios, sus negocios podrían permanecer en el tiempo.

\section{REFERENCIAS}

Ansoff, I., La Estrategia de la empresa, 1ª Ed., 1-241, Universidad de Navarra, ISBN:84-7634-226-8, Navarra, España (1976)

Barrios-Hernández, K., Olivero-Vega, E., y Acosta-Prado, J., Capacidad dinámica de innovación en instituciones de educación superior, Revista Espacios, ISSN 0798 1015, 38 (1), 1-24 (2017)

Barrios-Hernández, K., Olivero-Vega, E., y otros veintitrés autores, Gestión del conocimiento y capacidad de innovación. Modelos, Sistemas y Aplicaciones, $1^{\text {a }}$ Ed.,1-345, Universidad Simón Bolívar, ISBN 978-958-5430-05-1, Barranquilla, Colombia (2017)

Barrios-Hernández, K., y Olivero-Vega, E., Relación universidad-empresa-estado. Un análisis desde las instituciones de educación superior de Barranquilla-Colombia, para el desarrollo de su capacidad de innovación, http://dx.doi.org/10.4067/S0718-50062020000200021, Formación Universitaria, 13(2), 21-28 (2020)

Bohrnstedt, G.W., Evaluación de la confiabilidad y validez en la medición de actitudes, $1^{\text {a }}$ Ed., 103-127, Trillas, ISBN: 978-080-3915-95-4, Distrito Federal, México (1976)

Campo-Ternera, L., Amar-Sepúlveda, P., Olivero-Vega, E., y Huguett-Herazo, S., Emprendimiento e innovación como motor del desarrollo sostenible: Estudio bibliométrico (2006- 2016), https://doi.org/10.31876/rcs.v24i4.24907, Revista de Ciencias Sociales, 24(4), 26-36 (2018)

Cantillón, R., The circulation and exchange of goods and mechandise, $1^{\text {a }}$ Ed., 1-41, In H. Higgs, Essai sur la Nature du Commerce en Général, Londres, Inglaterra (1775)

Cárdenas, J., Guzmán, A., Sánchez, C., y Vanegas, J. D., ¿Qué se crea al fomentar el emprendimiento? Los principales impactos de la formación en este campo, http://dx.doi.org/10.12804/rev.univ.empresa.28.2015.08, Universidad y Empresa, 17(28), 173-190 (2015)

Correa, J., Ramírez, L., y Castaño, C., Modelo de Gestión financiera integral para Mipymes en Colombia, Contaduría Universidad de Antioquia, ISSN 0120-4203, 55 (1),187-201 (2009)

Consuegra, I., Barrios-Hernández, K., Contreras-Salinas, J., y Orozco-Acosta, E.E., Unidad de apoyo al microempresario: hacia la construcción de microempresas sostenibles en Barranquilla, $1^{\text {a }}$ Ed., 1-47, Universidad Simón Bolívar, ISBN 978-958-8930-79-4, Barranquilla, Colombia (2017)

Davis, S., y Meyer, C., The speed of change in the connected economy, $1^{\text {a }}$ Ed., 1-288, Grand Central Publishing, ISBN: 978-044-6675-33-8, Cambridge, Estados Unidos (1998)

Duarte, T., y Ruíz-Tibana, M., Emprendimiento, una opción para el desarrollo, Scientia Et Technica, ISSN: 0122-1701, 15(43), 326-331 (2009)

George, D., y Mallery, P., SPSS/PC+ Step by step. A simple guide and reference, 4th ed., Belmont Wadsworth Publishing, ISBN: 978-013-4320-25-0, Boston, Estados Unidos (1995)

Gutiérrez, J., Díaz, J., y Sulbarán, M., Emprendimiento como fuente de desarrollo de la empresa familiar. Algunas reflexiones sobre Venezuela, Revista de Ciencias Sociales, ISSN 2477-9431, 12(4), 98-107 (2017)

Hattie, J., Methodology Review: Assessing unidimensionality of tests and items, https://doi.org/10.1177/014662168500900204, Applied Psychological Measurement, 9, 139-164 (1985)

Kahn, J.H., Factor analysis in Counseling Psychology research, training and practice, https://doi.org 10.1177/0011000006286347, The Counseling Psychologist, 34, 1-36 (2006)

Kantis, H., y Angelelli, P., Los ecosistemas de emprendimiento en America Latina frente al Covid-19, Banco Interamericano de Desarrollo, https://www.iadb.org/es (2021)

Kreiser, P., Marino, L., Dickson, P., y Weaver, K., Cultural Influences on Entrepreneurial Orientation: The Impact of National Culture on Risk Taking and Proactiveness in SMEs, http://doi.org.10.1111/j.1540-6520.2010.00396, Entrepreneurship Theory and Practice, 34(5), 959-984 (2010)

Norman, A., Importance of financial education in making informed decision on spending, Academic Journal, ISSN 2006 9812, 2(10), 199-207 (2010)

MacCallum, R.C., Specification searches in covariance structure modelling, https://doi.org/10.1037/0033-

2909.100.1.107, Psychological Bulletin, 20, 100-107 (1986) 
Osterwalder, A., y Pigneur, Y., Generación de modelos de negocio. 14ª Ed., 1-282, John Wiley y Sons. Hoboken, ISBN 978-84-234-2799-4, New Jersey, Estados Unidos (2010)

Portafolio., Constitución de empresas en principales ciudades de Colombia en 2020, https://www.portafolio.co (2021)

Romero, J., Fonseca, G., y Blanco, F., Análisis y evaluación de la educación financiera en Boyacá, 1ªd, 1-157, Universidad Pedagógica y Tecnológica de Colombia, ISBN 978-958-6604-8-40 Tunja, Colombia, (2021)

Salinas-Ramos, F., y Osorio-Bayter, L., Emprendimiento y Economía Social: oportunidades y efectos en una sociedad en transformación. CIRIEC-España, http://doi.org/10.1016/S0737-6782(96)90159-9, Revista de Economía Pública, Social y Cooperativa, (75), 128-151 (2012)

Schumpeter, J.A., Capitalism, Socialism, and Democracy, Harper y Brothers, ISBN 978-161-7208-6-83 New York, Estados Unidos, (1942)

Teece, D., Dynamic Capabilities y Strategic Management. Organizing for Innovation an Growth, Oxford University Press Inc, ISBN 978-019-9691-90-6 New York, Estados Unidos, (2009)

Terjesen, S., y Hessels, J., Varieties of export-oriented entrepreneurship in Asia, http://doi.org/10.1007/s10490-0099138-3, Asia Pac J Manag, 26, 537-561(2009)

Valls, N., Villa, A., Martínez, S., y Hernando, A., Emprendimiento social Juvenil. 18 buenas prácticas, https://observatoritercersector.org/ (2009) 